\title{
Discharge of soft and hard grains and their mixtures from 2D silos
}

\author{
Jing Wang ${ }^{1}$, Kirsten Harth $^{1}$, Ralf Stannarius ${ }^{1, *}$ Bo Fan ${ }^{2}$, and Tamás Börzsönyi ${ }^{2}$ \\ ${ }^{1}$ Institute of Physics, Otto von Guericke University, Magdeburg, Germany \\ ${ }^{2}$ Institute for Solid State Physics and Optics, Wigner Research Center for Physics, Budapest, Hungary
}

\begin{abstract}
The outflow characteristics of hard grains from containers with narrow basal openings have been extensively studied. Recently, it was shown that soft, low-frictional grains can behave qualitatively different from the behavior of rigid grains. We compare experimentally the discharge of monodisperse hard spheres, soft spheres and mixtures of both from a quasi-twodimensional (2D) silo. The experiments demonstrate the remarkable consequences of the addition of few hard particles to a soft particle ensemble, as well as the gradual transition between the two limiting cases of pure one-component materials.
\end{abstract}

\section{Introduction}

Silos and hoppers have been used for centuries to store granular material in agriculture, chemical industry, construction industry and various other industrial branches. The discharge of material is conveniently realized by openings at the bottom, exploiting gravitational forces. Nevertheless, theories that quantitatively predict the outflow behavior of such materials through orifices at the container bottom are much less well developed than for liquids. One frequently encountered problem is congestion of the orifice, socalled clogging, even when the opening is much wider than the average grain size. Empirical formulae reasonably well describe outflow rates (e. g. $[1,2])$ and clogging statistics (e. g. [3-5]) for hard spheres, and a 'microscopic' model for the clogging behavior has been proposed [6]. For hard spheres, clogs can form when the orifice radius is smaller than approximately 5 particle diameters, and these clogs need to be dissolved mechanically.

The situation changes considerably when grains are soft and slippery [7-10]. With soft (elastic modulus of the order of $1 / 10$ of the bottom pressure in the silo), slippery (friction coefficient well below 0.1 ) grains, continuous outflow is found even through orifices with roughly twice the grain diameter. Below that, one can find intermittent flows and clogs that dissolve spontaneously $[8,9]$. The outflow rate has a pronounced fill height dependence and the bottom pressure does not saturate even when the fill height is much larger than the silo width $[8,10]$. Stagnant zones at the bottom are often absent. Some of these features are primarily related to the low friction coefficient, some to viscoelasticity, some to the combination of both.
Here we describe relevant aspects of the transition scenario from soft, low-frictional to rigid spheres in a quasi-2D silo, by studying mixtures of both grain types. Effects of the addition of small amounts of hard spheres to an ensemble of soft low-frictional spheres were explored recently [11]: The clogging frequency at small orifices is noticeably increased. The discharge rate within avalanches decreases remarkably. We extend this study to mixtures with a much higher hard particle content.

\section{Particles and experimental setup}
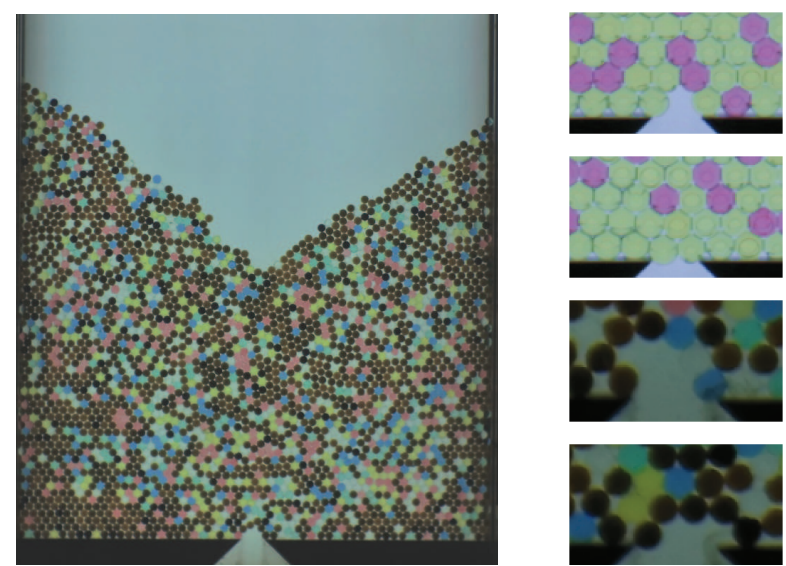

Figure 1. Left: Front view of the 2D silo containing a $50 \%: 50 \%$ mixture (M50) of hard (black) and soft (colored) spheres of the same size in a clogged state. $34 \mathrm{~cm}$ of the silo width are visible, the rest is hidden by aluminum rails at the sides. Right: Typical structures of clogs, top two images: pure HGS, $\rho=1.83(D=11 \mathrm{~mm})$; bottom two images: M50, $\rho=2.67(D=16 \mathrm{~mm})$.

*e-mail: ralf.stannarius@ovgu.de

A video is available at https://doi.org/10.48448/p4m1-d410

(C) The Authors, published by EDP Sciences. This is an open access article distributed under the terms of the Creative Commons Attribution License 4.0 
The setup consists of a container of $80 \mathrm{~cm}$ height, $40 \mathrm{~cm}$ width and slightly more than $6 \mathrm{~mm}$ depth (Fig. 1). A rectangular slit with variable width $D$ in the middle of the basal plate serves as outlet. The ratio of $D$ and the particle diameter $d$ is $\rho$.

The two components studied are hydrogel spheres (HGS) and airsoft beads (ASB), both with a comparable density of $\approx 1020 \mathrm{~kg} / \mathrm{m}^{3}$ and $6 \mathrm{~mm}$ diameter. The ASB have a friction coefficient of $\mu=0.3$, while that of the HGS is more than one order of magnitude smaller. Whereas the ASB can be considered rigid in our experiments, HGS have an elastic modulus of 50 to $100 \mathrm{kPa}$. They deform slightly under the pressure at the container bottom (of the order of a few $\mathrm{kPa}$ ). The HGS as well as the ASB are incompressible. M50 denotes a mixture containing 50\% ASB and $50 \%$ HGS. The full silo comprises about 10,000 grains. Since ASB and HGS have similar volumes, shapes and weights, we can single out the influences of frictional and elastic properties on silo discharge dynamics.

We combine two observation techniques. The discharged grains are collected in a container below the orifice and weighted. Simultaneously, videos of the transparent silo are recorded for the determination of the 2D flow field. Permanent clogs are destroyed by air flushes through the orifice which restart the flow. Air flushes were triggered manually when the flow stopped for at least 30 seconds (which is our definition for permanent clogs in this study). We note, however, that we cannot exclude that individual clogs can resolve spontaneously after more than 30 seconds. The reason for those non-permanent cessations of the outflow are the same that were described for pure soft grain at $\rho \approx 2[9]$.

\section{Experimental results}

\subsection{Silo discharge rate}

The outflow of hard spheres of diameter $d$ from a quasi-2D silo with a rectangular orifice of width $D$ and cell thickness $w$ can be described by Beverloo's equation [1], which we use here in the form

$$
\dot{m}=C \rho(D-k d)^{1.5} g^{0.5} w
$$

$C$ and $k$ are dimensionless constants, $w \approx d$ is the silo thickness, $\dot{m}$ is the mass discharged per time interval. At large orifice widths $(\rho>5)$, the samples behave qualitatively similar, but quantitatively different. At $\rho=5.83(D=35 \mathrm{~mm})$, for example, ASB flow with a constant rate of $\dot{m}=35.4 \mathrm{~g} / \mathrm{s}$ until the container is almost empty. Assuming the constant $k$ as 1.6 , we can calibrate the constant $C \approx 0.45$, which is expected to mainly depend on a filling fraction above the orifice. The pure HGS initially flow out with more than twice that rate, $\dot{m}=88 \mathrm{~g} / \mathrm{s}$. During discharge, this rate drops by about $20 \%$. The reason for the faster discharge of the soft spheres is the different flow structure in the silo. The central part of the HGS-filled silo is in continuous motion downward even well above the orifice, while the hard grains are repeatedly stopped in internal arches. They start to fall freely only near the outlet. This appears to be mainly a consequence of low-friction: Less gravitational energy is dissipated during the discharge.

Figure 2 shows examples of the discharge of ASB, HGS and the balanced mix M50 through a small orifice of $\rho=3(D=18 \mathrm{~mm})$. The HGS still run out continuously, initially with $\dot{m}=26 \mathrm{~g} / \mathrm{s}$. The M50 mixture, however, frequently clogs. In some cases these clogs dissolve spontaneously. The mean avalanche size is $58 \mathrm{~g}$ (roughly 500 grains), and also the initial discharge rate during avalanches is lowered considerably, to only $8.2 \mathrm{~g} / \mathrm{s}$. The pure ASB clog much more frequently, and all clogs are permanent. Mean avalanche sizes are down to $12 \mathrm{~g}$ (roughly 100 grains). This value may be slightly overestimated since the air flushes disturb the packing near the orifice. They also influence the outflow at the start of avalanches. We estimate a flow rate during avalanches of less than $6 \mathrm{~g} / \mathrm{s}$.

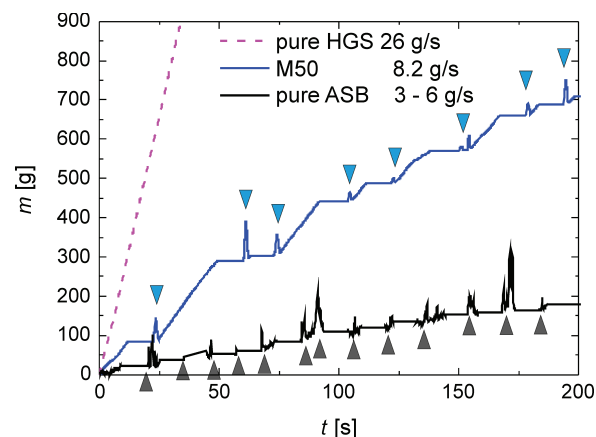

Figure 2. Discharges of ASB, HGS and the balanced mixture M50 through a $D=18 \mathrm{~mm}$ orifice $(\rho=3)$. Triangles mark air flushes. Plateaus of clogged states are clipped to $10 \mathrm{~s}$. Spikes are artifacts caused by the air flushes.

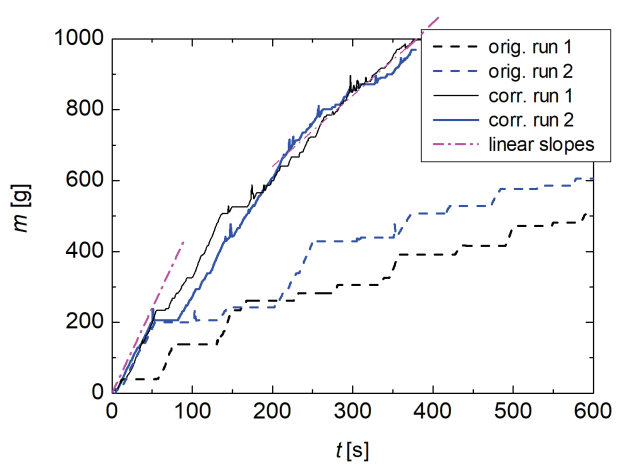

Figure 3. Two examples of discharge of the M50 mixture through a $\rho=2.67$ ( $D=16 \mathrm{~mm}$ opening), with the phases of permanent clogs eliminated. One can still see plateaus where the outflow stopped temporarily. The dash-dotted lines indicate the approximate outflow rates of the initial discharge $(m<200 \mathrm{~g})$ and the final phase $(m>800 \mathrm{~g})$.

Note that only geometric parameters enter Eq. (1). Nevertheless, even though the two materials studied 
here have similar sizes and shapes, the outflow rates during avalanches are quantitatively very different. The soft grains discharge faster, even when we clip all clogged phases. The explanation was given above.

An obvious feature not only of the pure HGS, but also of the mixtures (at least up to $60 \%$ ASB) is the fill-height dependent dynamics, regarding both clogging statistics and discharge rates at small $\rho$. This becomes obvious when we clip the permanently clogged states from the discharge curves (Fig. 3). The original curves (dashed) are shifted to the solid ones. Even with the permanent plateaus removed, the discharge rate between the clogs is fill-height dependent. The initial outflow rate at $\rho=2.67(D=16 \mathrm{~mm})$ during avalanches is about $4 \mathrm{~g} / \mathrm{s}$, while it drops monotonously to about $2 \mathrm{~g} / \mathrm{s}$ (dash-dotted lines). At that point, the fill level is still more than $100 \mathrm{~mm}$ above the orifice. It is obvious that Beverloo's original equation cannot be used unmodified to describe these mixtures. We attribute this feature primarily to the elastic properties of the grains: With sinking fill level, the pressure in the silo lowers, and the elastic distortions become correspondingly smaller and less relevant.

Similarly, the probability for permanent clogs increases with lowering silo content. Figure 4 shows the discharged masses until the $n$-th permanent clog. It is evident that at lower fill heights (larger discharged mass $m_{n}$ ), the mean avalanche sizes between successive clogs decrease. The presence of soft spheres in the mixture reduce the clogging probability as long as the fill height of the silo is high and the pressure at the silo bottom is large. With decreasing fill level, this pressure decreases and the elastic character of the HGS becomes less relevant. Consequently, the clogging probability raises and avalanches become shorter. For ASB the mean avalanche sizes show no trend with changing fill height.

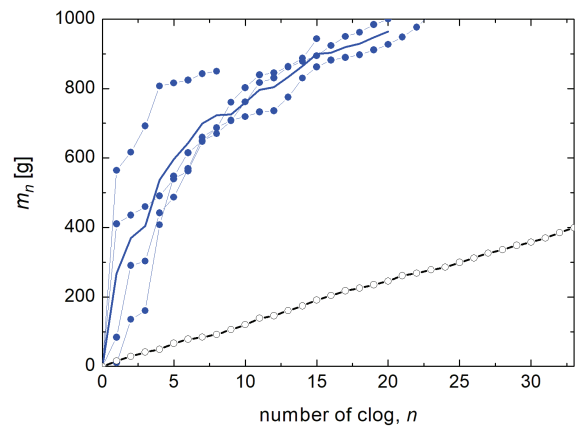

Figure 4. Cumulative plot of the discharged mass $m_{n}$ until the $n$-th permanent clog has formed, orifice size ratio $\rho=3$. Solid symbols: four individual runs with M50; thick line: average for M50; crosses: average for pure ASB. Pure HGS do not clog at all at this orifice width.

\subsection{Clog structure and stability}

Contrary to hard, frictional spheres in 2D silos, soft slippery grains and mixtures containing them show non-permanent congestions which spontaneously dissolve after some time [9]. Spontaneous unclogging occurs due to global rearrangements in the packing while the orifice is blocked: The packing structure compacts, causing increasing elastic deformations of soft grains at the bottom of the container. This leads finally to the breakage of blocking arches at the orifice. With pure HGS, permanent clogs occur only at small fill heights, or small orifice ratios $(\rho<2)$. For mixtures with up to $10 \%$ ASB, it was shown that permanent clogs are significantly more frequent [11]. In these studies, clogs were analyzed for $\rho \approx 2$. Most of them consisted of four spheres (Fig. 1) and they contained about twice as many hard grains than statistically expected from the mixture ratio.

In the present study of M50, we observe more permanent than temporary clogs. At the orifice size ratio of $\rho=3$, clogs consisted on average of 6 grains. The probability clogs containing more than one soft grain is very low. Statistically, we find a ratio of $7: 1$ hard to soft spheres forming stable clogs (see, e. g., Fig. 1 bottom right). Since both types are equally present in M50, the probability of ASB participating in a clog is larger by a factor of 6. Qualitatively, this finding agrees with the observation of much smaller avalanche sizes in pure ASB than in M50. The composition of a mixture also alters the structure of the clogging arches. For hard frictional spheres, they may even contain concave sections with dangling beads (see, e.g., Ref. [12]). In the M50 mixture, we also observe that the ratio of permanent to transient clogs increases statistically with lowering fill level. This indicates that the elastic deformability is the primary reason for spontaneous clog breakage. With lowering pressure in the granular bed, the HGS behave more less elastic and clogs tend to become persistent.

\subsection{Inner flow fields}

The flow inside the silo during avalanches is extracted from video imaging. A simple method to distinguish flowing from stagnant regions is the superposition of subsequent video images, as shown in Fig. 5. Blurred regions evidence motion of the grains (mainly downward) during the time interval of the overlays. Grains that remain in stagnant zones are clearly resolved. We show four mixture compositions with different shares of ASB. In the pure HGS sample (top left), the complete interior is in motion. Stagnant zones are practically absent. Well above the bottom (about one silo width high), the flow has plug-like character. This is primarily the consequence of the very low friction coefficient. The addition of up to $10 \%$ of ASB does not change this picture qualitatively. The M50, as well as the pure ASB, clearly show stagnant zones. In the flowing zones, the flow is converging towards the 
small orifice at the bottom. In both cases, the angles of the stagnant zones are very close to $60^{\circ}$. This is a consequence of the triangular lattices in these zones and the formation of narrow shear bands along the lattice planes. It is seen in Figs. 1 and 5 that the M50 mixtures slightly segregate during filling, even though we took particular care to avoid this. Hard grains tend to accumulate at the bottom and the lateral walls. In the zones participating in the flow, HGS are somewhat overrepresented.
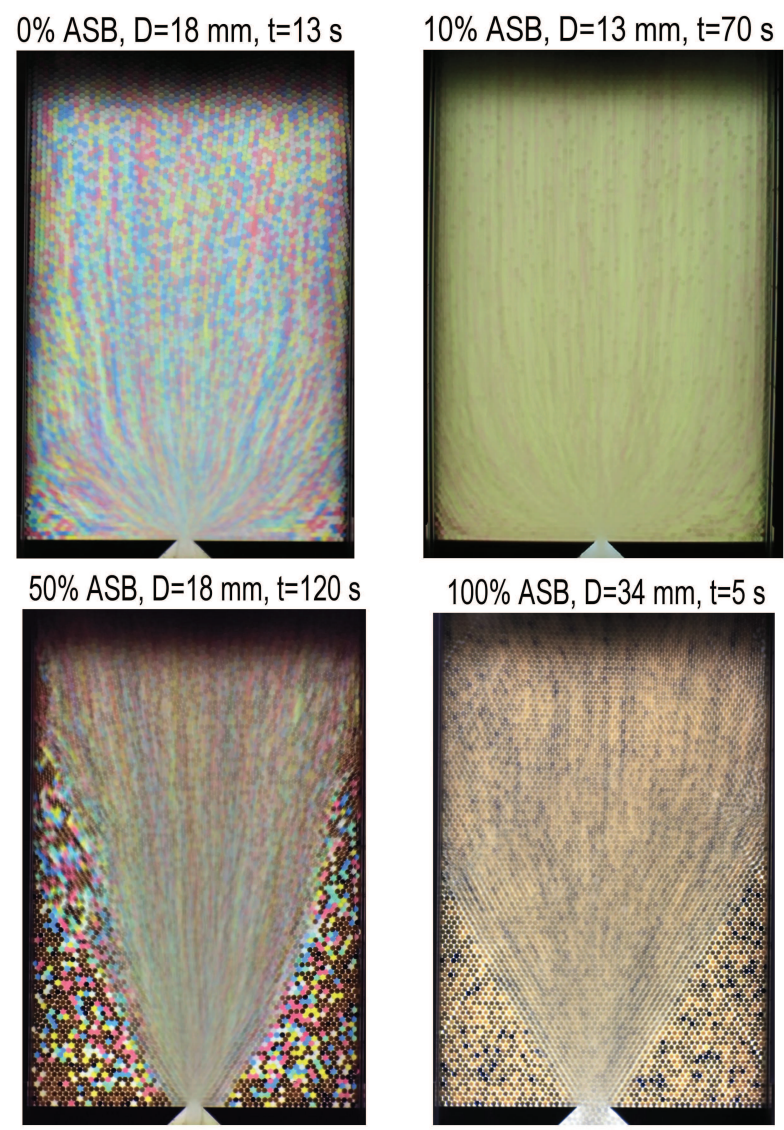

Figure 5. Superimposed images for a qualitative mapping of the internal flow field. In stagnant regions, the particles are clearly resolved, in flowing zones, they are motion blurred. The time intervals $t$ used for averaging were chosen such that both zones are clearly distinguishable. The choice of $t$ is not critical.

\section{Summary}

While the discharge of pure ensembles of soft, lowfrictional grains from a container with flat bottom involves the complete silo content, and the same applies for mixtures with few (up to $10 \%$ ) hard grains, funnel flow with stagnant zones is observed when the ratio of hard grains reaches $50 \%$ or more. The transition occurs at concentrations between $10 \%$ and $50 \%$ hard particles. This is primarily the consequence of the low friction of HGS.

Mixtures, even when they contain $50 \%$ hard, frictional grains produce non-permanent congestions of the outflow that dissolve spontaneously. Those can last for dozens of seconds. They are caused by the viscoelastic character of the HGS. Hard, frictional grains are highly over-represented in clogging structures in the mixtures. In particular, the permanent clogs are usually formed by the hard component, unless the silo fill height has dropped to few centimeters height. Then, soft grains are more likely to participate in stable clogs. The clogging probability of M50 mixtures increases substantially with lowering fill height of the silo. This is in agreement with the behavior of pure HGS ensembles and mixtures with few percent of hard, frictional ASB added.

With lower fill height and thus lower pressure near the orifice, not only the clogging probability increases (smaller mean avalanche sizes) but also, the discharge rate during avalanches decreases. Beverloo's equation can no longer describe the outflow of these mixtures satisfactorily. We assume that this is primarily the consequence of the low friction. This feature leads to less dissipation of the released gravitational energy during the discharge. Further experiments with frictional elastic and low-frictional rigid grains are needed to check these hypotheses.

This project received funding from the European Union's Horizon 2020 research and innovation program under the Marie Sklodowska Curie grant agreement No 812638 .

\section{References}

[1] W.A. Beverloo, H.A. Leniger, J.J. Van de Velde, Chem. Eng. Sci. 15, 260 (1961)

[2] C. Mankoc, A. Janda, R. Arévalo, J.M. Pastor, I. Zuriguel, A. Garcimartín, M. D., Granul. Matter 9, 407 (2007)

[3] I. Zuriguel, A. Garcimartín, D. Maza, L.A. Pugnaloni, J.M. Pastor, Phys. Rev. E 71, 051303 (2005)

[4] A. Janda, I. Zuriguel, A. Garcimartín, L.A. Pugnaloni, D. Maza, EPL 84, 44002 (2008)

[5] I. Zuriguel, Papers Phys. 6, 060014 (2014)

[6] C.C. Thomas, D.J. Durian, Phys. Rev. Lett. 114, 178001 (2015)

[7] X. Hong, M. Kohne, M. Morrell, H. Wang, E.R. Weeks, Phys. Rev. E 96, 062605 (2017)

[8] A. Ashour, T. Trittel, T. Börzsönyi, R. Stannarius, Phys. Rev. Fluids 2, 123302 (2017)

[9] K. Harth, J. Wang, T. Börzsönyi, R. Stannarius, Soft Matter 16, 8013 (2020)

[10] T. Pongó, J. Török, V. Stiga, B. Szabó, S. Lévay, R. Stannarius, R.C. Hidalgo, T. Börzsönyi, New J. Phys. 23, 023001 (2021)

[11] J. Wang, B. Fan, T. Pongó, K. Harth, T. Trittel, R. Stannarius, M. Illig, T. Börzsönyi, R. Cruz Hidalgo, Soft Matter, DOI:10.1039/D0SM01887B (2020)

[12] C. Lozano, G. Lumay, I. Zuriguel, R.C. Hidalgo, A.Garcimartín, Phys. Rev. Lett. 109, 068001 (2012) 